\title{
RAPID DETECTION OF FETAL ANEUPLOIDIES BY QUANTITATIVE FLUORESCENT-POLYMERASE CHAIN REACTION FOR PRENATAL DIAGNOSIS IN THE TURKISH POPULATION
}

Guzel AI ${ }^{1, *},{ }^{*}$ Yilmaz $\mathrm{MB}^{2, *}$, "Demirhan $\mathrm{O}^{2}$, Pazarbasi $\mathrm{A}^{2}$, Kocaturk-Sel $\mathrm{S}^{2}$, Erkoc $\mathrm{MA}^{2}$, Inandiklioglu $\mathrm{N}^{2}$, Ozgunen $\mathrm{FT}^{3}$, Sariturk $\mathrm{C}^{4}$

\#*These authors contributed equally to the preparation of this manuscript.

*Corresponding Author: Associate Professor Ali Irfan Guzel, Department of Medical Biology and Genetics, Faculty of Medicine, Rize University, 53100, Rize, Turkey; Tel.: +90-464-212-30-09; Fax: +90464-212-30-15; E-mail: aliirfanguzel@hotmail.com

\section{ABSTRACT}

Prenatal diagnosis is testing for diseases or conditions in a fetus or embryo before it is born. It employs a variety of techniques to determine the health and condition of an unborn fetus. The main goal of this process is to perform prenatal diagnosis at the earliest possible stage of gestation. In this regard, quantitative fluorescent-polymerase chain reaction (QF-PCR), a novel technique that is fast and reliable, was employed to detect aneuploidies (13, $18,21, \mathrm{X}$ and $\mathrm{Y}$ ) without the need of the time-consuming culturing process. The QF-PCR method can detect five different chromosome aneuploidies with $98.6 \%$ accuracy. In this study, 1874 amniotic fluid samples of pregnant subjects, who were referred to the Department of Medical Biology and Genetics, Adana, Turkey (molecular biology section), were analyzed with the QF-PCR technique by employing 27 short tandem repeat (STR) markers to detect chromosomes 13, 18, 21, $\mathrm{X}$ and $\mathrm{Y}$ aneuploidies. We detected 31 subjects $(1.7 \%)$ with aneuploidies or euploidies out of the 1874 subjects. The average age of the pregnant subjects was 32 (range: 14-49).

Department of Medical Biology and Genetics, Faculty of Medicine, Rize University, Rize, Turkey

2 Department of Medical Biology and Genetics, Faculty of Medicine, Çukurova University, Adana, Turkey

3 Department of Obstetrics and Gynecology, Faculty of Medicine, Çukurova University, Adana, Turkey

4 Department of Biostatistics, Faculty of Medicine, Çukurova University, Adana, Turkey
Abnormal karyotypes detected were as follows: 47,XX,+21 (19.4\%, 6/31), 47,XY,+21 (48.4\%, $15 / 31), 48, \mathrm{XXX},+21(3.2 \%, 1 / 31), 69, \mathrm{XXX}(3.2 \%$, $1 / 31), 47, \mathrm{XY},+13(3.2 \%, 1 / 31), 47, \mathrm{XXY}(9.6 \%$, $3 / 31), 47, \operatorname{XXX}(9.6 \%, 3 / 31)$ and $45, X(3.2 \%, 1 / 31)$. Moreover, some STR markers were found to be more specific to the Turkish population. In conclusion, QF-PCR can be regarded as an alternative method of conventional cytogenetic analysis as it is a rapid and reliable method; however, in most cases it is required to be supported or validated with conventional cytogenetic karyotyping and some STR markers employed for QF-PCR can be more informative for a given population.

Keywords: Aneuploidy, Cytogenetics, Euploidy, Prenatal diagnosis, Quantitative fluorescent-polymerase chain reaction (QF-PCR), Trisomy.

\section{INTRODUCTION}

Pregnant women who are at increased risk of chromosome abnormalities (usually due to maternal age, altered serum metabolites, or ultrasound abnormalities in fetus), undergo invasive sampling, either amniotic fluid (AF), chorionic villus (CV) or, rarely, fetal blood (FB). Cells from these samples are used either for full karyotype analysis or for DNA extraction, which would be used in molecular studies to detect numerical chromosome anomalies or specific gene mutations by quantitative fluorescent-polymerase chain reaction (QF-PCR) or real time PCR, respectively [1]. Prenatal diagnosis of common 
chromosomal aneuploidies is routinely achieved by standard cytogenetic techniques. In these procedures, fetal cells must be cultured for up to 2 (or more) weeks before analysis. Therefore, the duration of these processes is the major disadvantage of these techniques. A rapid diagnosis is of paramount importance in the case of a fetus with abnormal ultrasound findings, which may, in most cases, suggest an aneuploidy for chromosomes $21,18,13$, or $\mathrm{X}$ (the most common chromosomal aneuploidies) [2]. In the early 1990s, fluorescence in situ hybridization (FISH) and, more recently, QF-PCR, has been introduced to the field of prenatal diagnosis to overcome the need of fetal cell culturing that also allows rapid diagnosis of the most common chromosomal anomalies, thereby enabling physicians to manage the pregnancy of an abnormal fetus at an earlier time of gestation [3]. The QF-PCR method is based on PCR amplification of selected chromosome-specific short tandem repeat (STR) markers $[4,5]$. During the amplification process, a fluorochrome is incorporated into the amplified products that can be visualized and quantified accurately as peaks on automated DNA scanners. Normal heterozygous subjects for a specific chromosome are expected to have two peaks where the area below the peaks are at a 1:1 ratio. The trisomies, however, are visualized either as three peaks with a $1: 1: 1$ ratio (trisomic triallelic subjects) or as two peaks with a 2:1 ratio (trisomic diallelic subjects) when comparing the peak areas of a specific STR marker [4]. Parental origin of the aneuploidy can also be deduced by comparing the fetal allele sizes in base pairs with parental allele sizes in base pairs $[4,6,7]$. Quantitative fluorescent-PCR is increasingly being considered and proposed as a complementary investigation, or even as an alternative to conventional cytogenetic analysis for prenatal diagnosis in most clinical settings [8-10]. In summary, we report the results of multiplex (employing two or more sets of primers in one reaction condition) QF-PCR applied to 1874 pregnancies for the detection of the most prevalent autosomal and sex chromosome aneuploidies or euploidies.

\section{MATERIALS AND METHODS}

The majority of prenatal samples (98.99\%) were AF (the investigated pregnancies were from the South Anatolia region of Turkey) $(n=1874)$, collected between 14 and 26 weeks of gestation for our routine diagnostic tests at the Department of Medical Biology and Genetics, Faculty of Medicine, Çukurova University, Adana, Turkey. A total of four FB samples $(0.2 \%)$ and 15 fetal tissues (FT) from aborted fetuses $(0.8 \%)$ were also investigated. The most frequent indications for QF-PCR diagnosis of common chromosome aneuploidies were advanced maternal age, abnormal serum a-fetoprotein (AFP) levels and abnormal ultrasound findings. All the women who participated were informed about the advantages and limitations of the assay. Some AF samples that were dark in color instead of bright transparent yellow or contaminated with blood, were treated with distilled water in order to wash away possible maternal cell contamination (MCC). As a result of $\mathrm{MCC}$, if additional peaks were observed in the electrophoretograms, these suspected samples were tested simultaneously with corresponding maternal blood DNA samples to detect fetal and maternal STR patterns by comparing them to each other. All trisomic samples were validated with conventional karyotype analysis following QF-PCR testing. DNA extraction for QF-PCR was performed on AF, FB and FT samples by incubating cell pellets with InstaGeneTM Matrix (Bio-Rad Laboratories, Hercules, CA, USA). The QF-PCR amplifications were performed using AneufastTM (Molgentix SL, Barcelona, Spain) trisomy detection kit that included dNTPs, Hot Start Taq DNA polymerase and fluorescently-labeled primers for 27 STR markers (Table 1) located on chromosomes 13, 18, 21, X, and Y, and primer sets for amelogenin (AMXY) gene and sexdetermining region $\mathrm{Y}$ (SRY) regions, which were used for the detection of fetal sex in six different multiplex (employing two or more sets of primers in one reaction condition) PCR mixtures containing specific primers for the chromosome of interest STR regions in an optimized reaction mixture.

Reactions were prepared by combining $10 \mu \mathrm{L}$ of the STR/PCR mixtures (referred as S1 and S2, which contain primer sets for the STR regions of chromosomes 13, 18, 21, X and Y), 5-10 ng of amniotic cell DNA and PCR-grade water up to a total volume of $15 \mu \mathrm{L}$ and thermal cycled $(15 \mathrm{~min}$. at $95^{\circ} \mathrm{C}$ followed by 25 cycles of 40 seconds at $95^{\circ} \mathrm{C}$, 90 seconds at $60^{\circ} \mathrm{C}$ and 40 seconds at $72^{\circ} \mathrm{C}$, with a final extention at $60^{\circ} \mathrm{C}$ for $30 \mathrm{~min}$.) according to the manufacturer's protocol. The QF-PCR products [1.5 mL from each of the two main mixes (S1 and 
Table 1: STR markers used for QF-PCR detection of chromosomes 13, 18, 21, X and Y copy numbers.

\begin{tabular}{|c|c|c|c|}
\hline Marker & Label & Chromosome Location & Allele Ranges (bp) \\
\hline AMXY & 6-Fam & Xp22.1-22.31-Yp11.2 & X: 104; Y: 109 \\
\hline SRY & 6-Fam & Yp11.2 & Y: 463 \\
\hline X22 & 6-Fam & Xp28 Yq (PAR2) & $189-253$ \\
\hline DXYS218 & PET & Xp22.32 Yp11.3 (PAR1) & $266-294$ \\
\hline HPRT & 6-Fam & $\mathrm{Xq} 26.1$ & $268-313$ \\
\hline DXS6803 & $\mathrm{VIC}$ & $\mathrm{Xp} 12-\mathrm{Xq} 21.33$ & $106-128$ \\
\hline DXS6809 & VIC & $\mathrm{Xq}$ & $238-274$ \\
\hline DXS8377 & NED & $\mathrm{Xq} 28$ & $166-211$ \\
\hline SBMA & VIC & $\mathrm{Xq11.2-Xq12}$ & $166-211$ \\
\hline D21S1414 & 6-Fam & $21 \mathrm{q} 21$ & $328-443$ \\
\hline D21S1411 & VIC & $21 \mathrm{q} 22.3$ & $246-319$ \\
\hline D21S1446 & PET & 21q22.3-ter & $200-228$ \\
\hline D21S1437 & PET & $21 \mathrm{q} 21.1$ & $120-144$ \\
\hline D21S1008 & 6-Fam & $21 \mathrm{q} 22.1$ & $196-220$ \\
\hline D21S1412 & 6-Fam & $21 \mathrm{q} 22.2$ & $384-418$ \\
\hline D21S1435 & VIC & $21 \mathrm{q} 21$ & $142-188$ \\
\hline D18S391 & VIC & 18 pter-1p11.22 & $144-168$ \\
\hline D18S390 & VIC & $18 \mathrm{q} 22.2$ & $198-430$ \\
\hline D18S535 & NED & $18 \mathrm{q} 12.2$ & $126-156$ \\
\hline D18S386 & NED & $18 \mathrm{q} 22.1$ & $319-387$ \\
\hline D18S858 & PET & $18 \mathrm{q} 21.1$ & $186-204$ \\
\hline D18S499 & 6-Fam & $18 \mathrm{q} 21.32-\mathrm{q} 21.31$ & $386-408$ \\
\hline D18S1002 & 6-Fam & $18 \mathrm{q} 11.2$ & $122-142$ \\
\hline D13S631 & VIC & $13 q 31-32$ & $192-218$ \\
\hline D13S634 & VIC & $13 \mathrm{q} 14.3$ & $460-500$ \\
\hline D13S258 & NED & $13 q 21$ & $230-281$ \\
\hline D13S305 & PET & $13 q 12.1-13 q 14.1$ & $426-458$ \\
\hline D13S628 & 6-Fam & $13 q 31-q 32$ & $436-464$ \\
\hline D13S742 & VIC & $13 q 12.12$ & $254-274$ \\
\hline
\end{tabular}

S2)] were added into $40 \mu \mathrm{L} \mathrm{Hi}^{-D{ }^{T M}}$ Formamide (Applied Biosystems, Foster City, CA, USA), containing $0.3 \mu \mathrm{L}$ of GeneScan ${ }^{\mathrm{TM}}-500 \mathrm{LIZ}^{\mathrm{TM}}$ (Applied Biosystems) used as a size standard. After denaturation at $95^{\circ} \mathrm{C}$ for $3 \mathrm{~min}$., the mixtures were allowed to cool down to $4^{\circ} \mathrm{C}$ and then capillary electrophoresis was carried out on an ABI PRISM ${ }^{\mathrm{TM}} 3130$ Genetic Analyzer (Applied Biosystems) using a POP7 polymer.

Analysis of the results and calculations of the peak areas (peak area is the area under a specific STR peak) were performed using GeneMapper 4.0 software (Applied Biosystems). The criteria and guidelines for the determination of the QF-PCR results of normal and pathological cases were as follows: in normal individuals who were heterozygous for a given STR, the same amount of fluorescence was generated for both alleles. Therefore, the ratio between the fluorescent peak areas was 1:1 (ratios are calculated by dividing the area of the shorter allele in base pairs by the area of the longer one). In normal individuals who are homozygous (have the same repeat number) for a specific STR allele, the quantification is not possible so the marker is regarded as uninformative, and the ratio is therefore considered as 1 . In trisomic cases, the three copies 

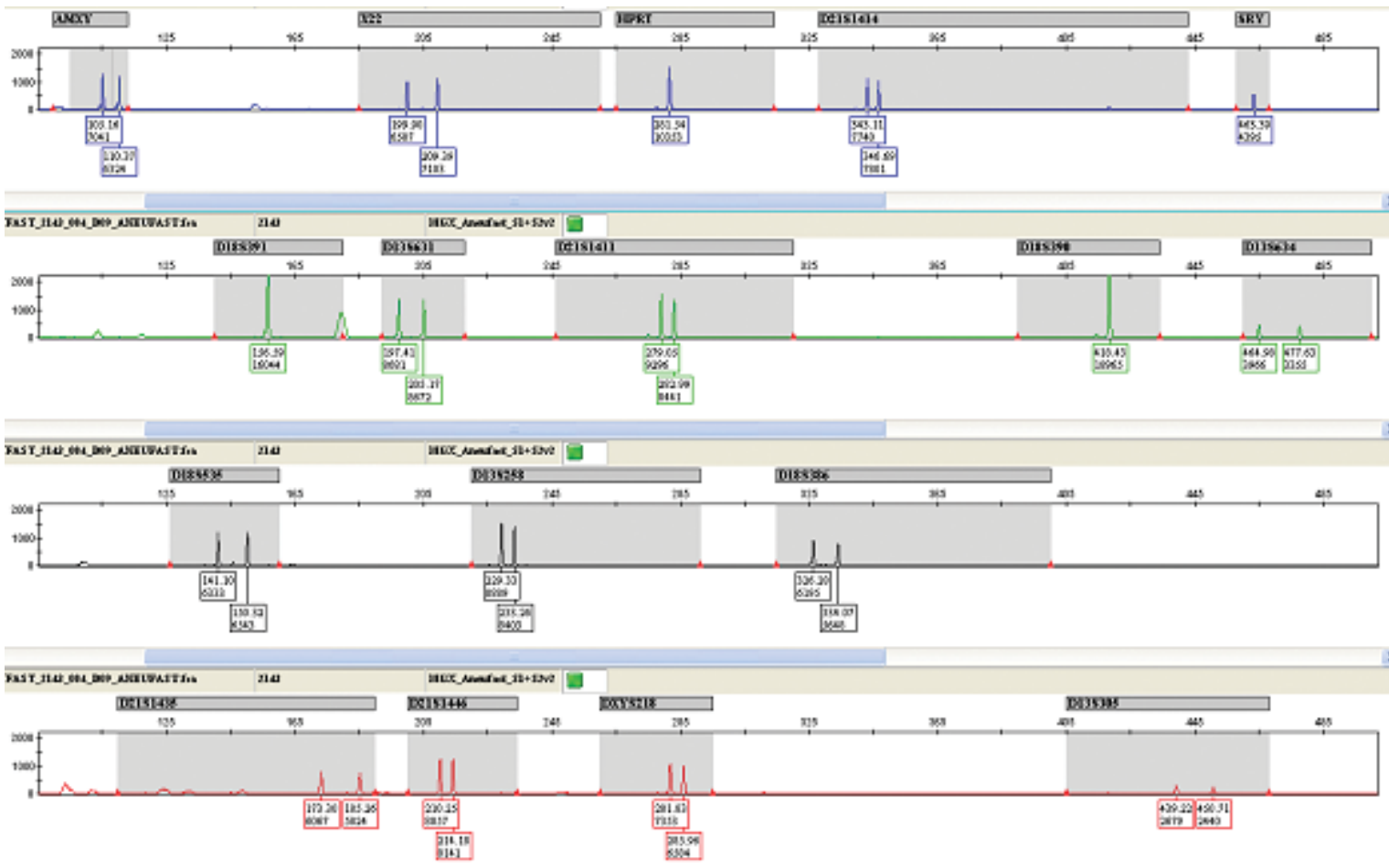

Figure 1. Electrophoregram of an individual diagnosed as normal for chromosomes 13,18, 21, X and Y. The ratio of peak areas that are the values in square boxes (upper value denotes the base pair and the bottom one corresponds to the area below the peak). These peak areas are used for comparing different alleles such as in AMXY, there are two peaks: the first one is $105.16 \mathrm{bp}$ long and the area for this peak is 7041; the second one is $110.37 \mathrm{bp}$ long and the area for that peak is 6324 , so the ratio between two peak areas is 7041:6324, which is almost a 1:1 ratio and considered to be in the acceptable limits for the assay.

of a specific chromosome can be detected as a 1:1:1 ratio (trisomic triallelic) or as a 2:1 ratio (trisomic diallelic) patterns when their peak areas are compared. Assessment of a normal or trisomic copy number is concluded when at least two informative markers were detected for the chromosome of interest (Aneufast User Manual; Molgentix SL).

\section{RESULTS}

The distribution of 31 pathological cases out of 1874 samples were as follow: 47, XX, $+21(19.4 \%$, $6 / 31), \quad 47, X Y,+21 \quad(48.4 \%, 15 / 31) \quad 48, X X X,+21$ $(3.2 \%, 1 / 31), 69, \mathrm{XXX}(3,2 \%, 1 / 31), 47, \mathrm{XY},+13$ $(3.2 \%, 1 / 31), 47, \mathrm{XXY}(9.6 \%, 3 / 31), 47, \mathrm{XXX}(9.6 \%$, $3 / 31), 45, \mathrm{X}(3.2 \%, 1 / 31)$. The ratio of heterozygous fluorescent peak areas were close to $1: 1$ for normal cases (Figure 1) and 1:1:1 or 2:1 for aneuploidies of chromosome-specific STRs (Figure 2). At least two informative results for each chromosome-specific STRs were required to evaluate the prenatal diagnosis. Aneuploidic QF-PCR results were validated with cytogenetic analyses to complete the diagnosis. No false-positive results were observed, and all the pathological QF-PCR results were consistent with the cytogenetic analyses. Fetal sex and chromosome $\mathrm{X}$ and $\mathrm{Y}$ copy numbers were determined by amplification of the non polymorphic AMXY sequences (generates chromosome $\mathrm{X}$ - and $\mathrm{Y}$-specific products, differing 6 bp in length) and SRY, and other X-and Y-specific STRs (Table 1, Figure 1). These findings were also in agreement with the cytogenetic analysis in all cases.

In cases with uninformative results that have none or only one 1:1 ratio for a given chromosome, extra reaction mixture containing two or more additional STR primer sets specific for the chromosome of interest, were employed in order to obtain informative results. Maternal cell contamination was detected by evaluating peak patterns in heterozygous STRs as such, if there are three alleles and the sum of the peak areas of the two smaller alleles is equal to the largest peak area then the smaller of the two alleles is regarded as the maternal contaminant. 


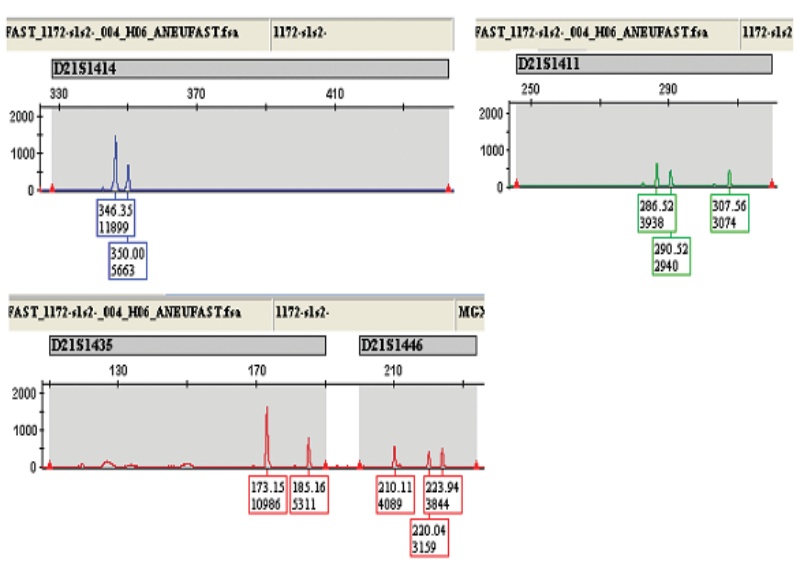

Figure 2. Electrophoregram of an individual diagnosed as trisomy for chromosome 21 (Down's syndrome). All chromosome 21-specific STRs have three copies with $2: 1,1: 1: 1,2: 1$ and $1: 1: 1$ ratios, consecutively.

The MCC sample results were clarified by comparing the fetal STRs with maternal STRs obtained from maternal blood DNA samples (Figure 3). The $\mathrm{X}$-specific product of the AMXY marker was found to be duplicated in three males producing fluorescent peak areas in the ratio of 2 (for the $\mathrm{X}$ chromosome) and 1 (for the Y chromosome), 47,XXY males. In all $\mathrm{X}$ chromosome-related aneuploidies (one 45,X, three $47, \mathrm{XXX}$, and three 47,XXY cases), the correct diagnosis was achieved by using all of the $\mathrm{X}$ chromosome-specific STR markers. The diagnosis of Turner's syndrome was based on the detection of a single QF-PCR product for all the X chromosomespecific polymorphic markers (data not shown).

Of all markers studied, X22 and DXYS218 had the highest frequency of heterozygosity, therefore, regarded as the most informative markers. As for chromosomes 13, 18 and 21, STR markers, D13S258, D18S386 and D211414-1411 had the highest heterozygosity, respectively (Figure 4). In terms of additional chromosome markers studied, which were employed to validate the results obtained with regular STR markers having one or none informative markers for a specific chromosome, STR markers, D13S742, D18S858, D211412 and DX8377, had the highest heterozygosity for chromosomes 13, 18, 21 and XY, respectively, in our study population (Figure 5).
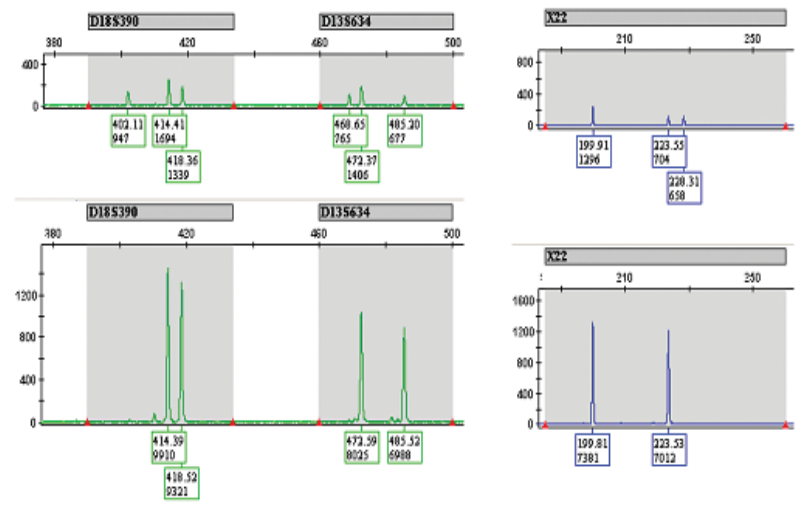

Figure 3. Electrophoregram of MCC in a sample [the upper row (fetus) in comparison with the lower row (mother)], for example in the D18S390 STR marker, both 414 and 418 bp long alleles come from mother in the fetus, implying a possible MCC, since only one allele from the mother should be present in the fetus.

\section{DISCUSSION}

The standard karyotype analysis implies the determination of number and structure of all 23 chromosome pairs. The most common chromosome anomalies, whose incidence increase with maternal age, are related to non disjunctional errors resulting in an extra copy or loss of one chromosome, either in all cells or in a mosaic state (11). These anomalies, however, have to be detected in a timely fashion during gestation, using rapid and reliable techniques such as QF-PCR, thus the pregnancy could be terminated (or not) earlier leading to less physical and emotional burdens to the mother and family.

Several groups have reported that, QF-PCR can be applied on different fetal samples (CVS, AF, FB, FT obtained after termination) and is suitable for detecting all major aneuploidies (4,12-15). Chromosome anomalies, however, can comprise the majority of all viable fetal aneuploidies, which has a slightly negative diagnostic effect (16). Rapid analysis of fetal chromosomes by QF-PCR: 1) reduces parental anxiety through the exclusion of numerical chromosome aberrations in fetuses, 2) provides rapid detection of major aneuploidies, particularly in fetuses suspected of carrying chromosome disorders on the basis of ultrasound or biochemical tests, 


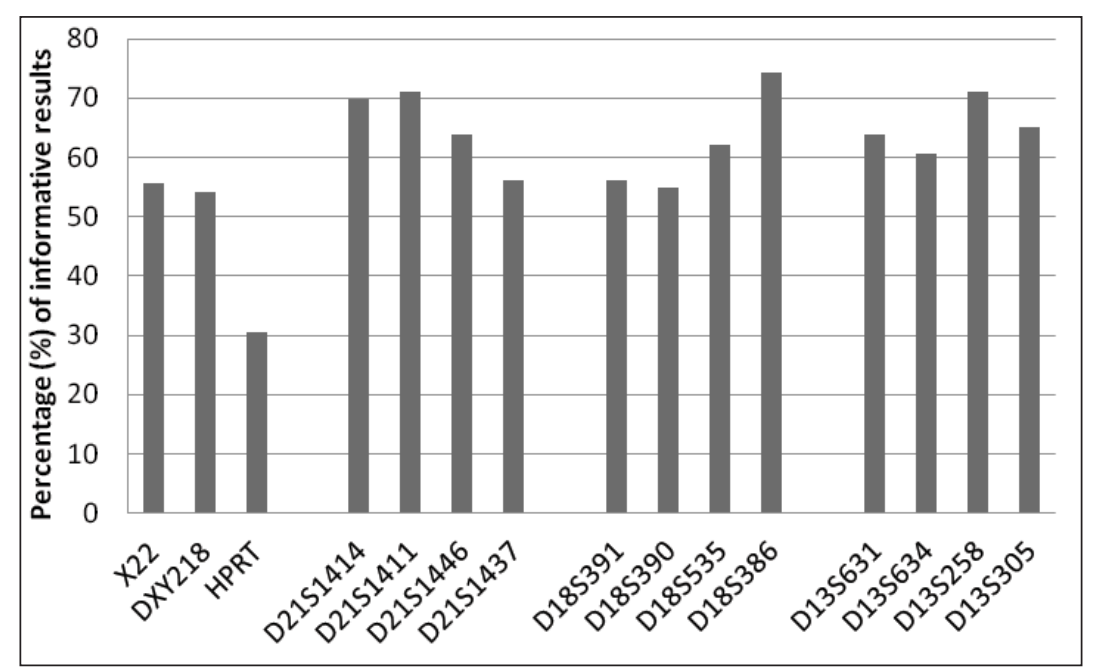

Figure 4. STR markers used for euploidy and aneuploidy detection of chromosomes 13, 18, 21 and XY and their percentage of informative results of the study group $(n=1874)$.

3) allows families to give a decision regarding termination of pregnancy at an earlier stage of gestation, when the result clearly indicates the presence of fetal chromosome disorder (6). Quantitative fluorescentPCR allowed the determination of the fetal origin of the sample and enabled us to eliminate MCC by comparing the alleles from the fetal sample with those from the mother. It is rapid (takes around 24 hours for diagnosis, depending on the number of samples studied), and a large number of samples can be processed simultaneously with lower costs (8-10,13).

According to ourfindings, STR markers D13S258 and D13S742 for chromosome 13; D18S386 and D18S858 for chromosome 18; D21S1414-1411 and D211412 for chromosome 21; X22, DXYS218 and DX8377 for gender chromosomes, had the highest heterozygosity ratio in our study population (Figures 4 and 5); therefore, these STR markers could be re- a)

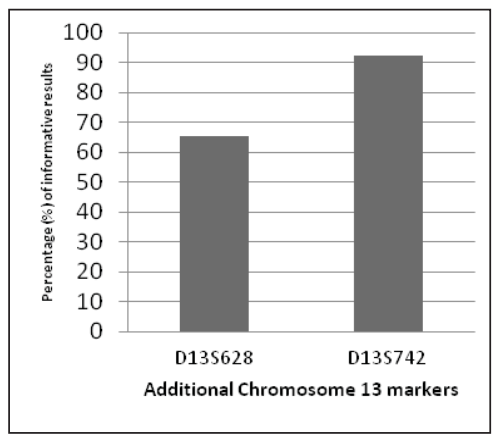

c)

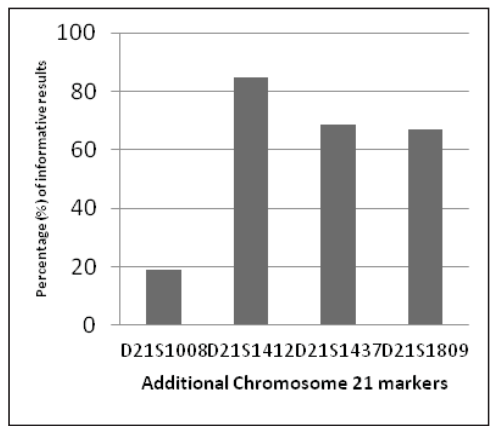

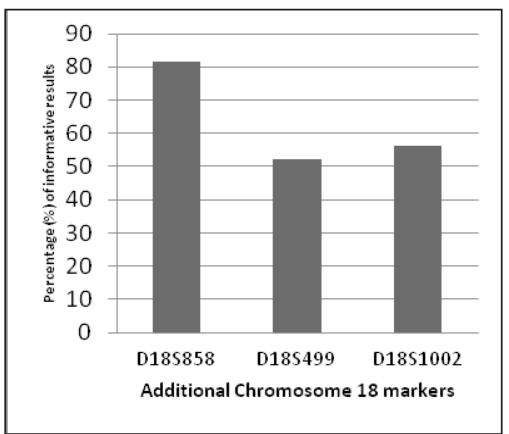

b)

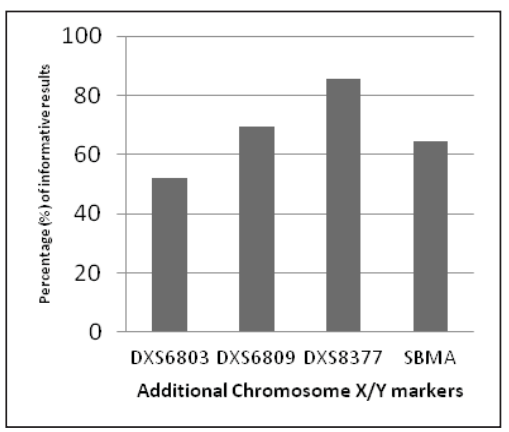

d)

Figure 5. Additional STR markers used for euploidy and aneuploidy detection of chromosomes 13 (a), 18 (b), 21 (c) and XY (d), respectively, and the percentage of informative results of each additional marker studied. 
garded as the most informative markers of common chromosome euploidies or aneuploidies. It is therefore plausible to employ these STR markers first to detect common chromosomal euploidies or aneuploidies in the Turkish population.

In conclusion, prenatal diagnosis of a fetus is very crucial, as this might affect the life of an individual and his/her family. In this regard, we focused on chromosomal euploidies and aneuploidies and how frequent these abnormalities arise during pregnancy. In our study, we observed that trisomies occurred in around $1.7 \%$ of all subjects tested and some STR markers we employed in our tests are better for detecting chromosomal aneuploidies, therefore regarded as more informative and should be the first to test in detecting numerical chromosomal aberrations. We also support the notion that QF-PCR is reliable, accurate, and robust, with no misdiagnoses for the non mosaic trisomies of chromosome 13, 18, 21, X and Y. However, the QF-PCR method can be used in conjunction with conventional cytogenetic techniques that are still regarded as gold standards.

\section{ACKNOWLEDGMENTS}

We are grateful to the Çukurova University Scientific Research Projects Unit (Adana, Turkey) for financially supporting this study.

\section{REFERENCES}

1. Lorda-Sanchez MB, Petersen F, Binkert M. A $48, \mathrm{XXY},+21$ Down syndrome patient with additional paternal X and maternal 21. Hum Genet. 1991; 87(1): 54-56.

2. Hassold TJ, Jacobs PA. Trisomy in man. Annu Rev Genet. 1984; 18(1): 69-97.

3. Von Eggeling F, Freytag M, Fahsold R, Horsthemke B, Claussen U. Rapid detection of trisomy 21 by quantitative PCR. Hum Genet. 1993; 91(6): 567-570.

4. Adinolfi M, Pertl B, Sherlock J. Rapid detection of aneuploidies by microsatellite and the quantitative fluo-rescent polymerase chain reaction. Prenat Diagn. 1997; 17(13): 1299-1311.
5. Mansfield ES, Kronick MN. Alternative labeling techniques for automated fluorescence based analysis of PCR products. Biotechniques. 1993; 15(2): 274-279.

6. Diego-Alvarez D, Ramos-Corrales C, Garcia-Hoyos M. Double trisomy in spontaneous miscarriages: cytogenetic and molecular approach. Hum Reprod. 2006; 21(4): 958-966.

7. Lamb NE, Freeman SB, Savage-Austin A. Susceptible chiasmate configurations on chromosome 21 predispose to non-disjunction in both maternal meiosis I and meiosis II. Nat Genet. 1996; 14(4): 400-405.

8. Grimshaw GM, Szczepura A, Hulten M. Evaluation of molecular tests for prenatal diagnosis of chromosome abnormalities. Health Technol Assess. 2003; 7(10): 1-77.

9. Leung WC, Lau ET, Lao TT, Tang MH. Rapid aneuploidy screening (FISH or QF-PCR): the changing scene in prenatal diagnosis? Expert Rev Mol Diagn. 2004; 4(3): 333-337.

10. Ogilvie CM. Prenatal diagnosis for chromosome abnormalities: past, present and future. Pathol Biol. 2003; 51(3): 156-160.

11. Spathas DH, Divane A, Maniatis GM, FergusonSmith ME, Ferguson-Smith MA. Prenatal detection of trisomy 21 in uncultured amniocytes by fluorescence in situ hybridization: a prospective study. Prenat Diagn. 1994; 14(11): 1049-1054.

12. Mann K, Ogilvie CM. Prenatal detection of chromosome disorders. Lancet. 2001; 358(9293): 1030-1031.

13. Pertl B, Kopp S, Kroisel PM. Rapid detection of chromosome aneuploidies by quantitative fluorescence PCR: first application on 247 chorionic villus samples. J Med Genet. 1999; 36(4): 300-303.

14. Rahil H, Solassol J, Philippe C, Lefort G, Jonveaux P. Rapid detection of common autosomal aneuploidies by quantitative fluorescent PCR on uncultured amniocytes. Eur J Hum Genet. 2002; 10(8): 462-466.

15. Verma L, MacDonald F, Leedham P, McConachie M, Dhanjal S, Hultén M. Rapid and simple prenatal diagnosis of Down's syndrome. Lancet. 1998; 352(9121): 9-12.

16. Sherlock J, Cirigliano V, Petrou M, Tutschek B, Adinolfi M. Assessment of diagnostic quantitative fluorescent multiplex polymerase chain reaction assays performed on single cells. Ann Hum Genet. 1998; 62(1): 9-23. 